\title{
DYSPLASTIC WHITE MATTER LESIONS IN PATIENT WITH NEUROFIBROMATOSIS 1
}

\author{
P. Amaresh Reddy¹, Amit Agrawal ${ }^{3}$, V. Umamaheshwar Reddy², P. Radharani' ${ }^{1}$, \\ Sahith Reddy ${ }^{2}$ \\ ${ }^{1}$ Department of Endocrinology, Narayana Medical College Hospital, Chinthareddypalem, Nellore, \\ Andhra Pradesh (India) \\ ${ }^{2}$ Department of Radiology, Narayana Medical College Hospital, Chinthareddypalem, Nellore, \\ Andhra Pradesh (India) \\ ${ }^{3}$ Department of Neurosurgery, Narayana Medical College Hospital, Chinthareddypalem, Nellore, \\ Andhra Pradesh (India)
}

\begin{abstract}
Dysplastic white matter lesions/unidentified bright objects /Foci of abnormal signal intensities (FASi's) in brain MRI are the commonest intracranial abnormality with Neurofibromatosis 1 seen in approximately $70-75 \%$ of patients. They are usually multiple, small in size and are typically located in globus pallidus, brainstem, centrum semiovale, thalamus, internal capsule, corpus callosum, and cerebellum. Although clinically silent, patients can present with reduced attention span however neuropsychological functioning of these lesions depends upon the region involved. NF1 lesions should be kept as differential for any hyperintense lesion in basal ganglia and caution is advised not to confuse these lesions with malignant lesions like gliomas as biopsies from these lesions showed benign etiology. Parental counselling regarding the prognosis is very important to alleviate unnecessary apprehension. Interval follow-up is advised for large lesions causing mass effect, showing contrast enhancement or when lesions are located in optic pathway.
\end{abstract}

Keywords: Neurofibromatosis-1, dysplastic white matter lesions, unidentified bright objects, Foci of abnormal signal intensities, cognition

\section{INTRODUCTION}

Neurofibromatosis type 1 (NF1) is inherited chromosomal syndrome in which individuals develop multiple structural and functional brain abnormalities. (1-3) Dysplastic white matter/unidentified bright objects/Foci of abnormal signal intensities (FASi's) in brain MRI are the commonest intracranial abnormality with Neurofibromatosis 1 (NF1). (1, 4-6) They are seen in approximately $70-75 \%$ of patients with NF1, incidence is higher in children with optic gliomas. $(1,4)$ These lesions are rarely seen below 2 yrs of age and above $20 \mathrm{yrs}$. $(1,5)$ They appear at around 3yrs of age and increase in number and size up to twelve years, there onwards they spontaneously regress. $(1,4,7)$ Cognition and behavioral abnormalities have been attributed to these white matter lesions. $(5,8,9)$ We describe a case of NF1 who presented to us with cognition abnormalities and behavioral problems and on MRI we found dysplastic white matter lesions.

\section{CASE REPORT}

A 12 year old boy had been brought by his parents with complaints of poor school performance, short stature and history of not mingling with children of his own age. On general examination his vitals were stable; height of the child was low for 
his age, skin examination showed 9 café eu lait spots (0.6-1.3 cms size), few subcutaneous swellings over the body. His IQ was mildly low for his age. Skeletal survey with plain radiographs did not reveal any abnormality. Bone age was appropriate, CNS examination revealed normal power and reflexes. Ophthalmic examination was normal. Diagnosis of Neurofibromatosis type 1 was confirmed based on above findings. Thyroid hormone levels, Growth hormone levels were normal. MRI was advised to rule out central causes of short stature (Hypothalamopituitary axis) as well as to look for associated cerebral manifestations of NF1. MRI was performed on 3T GE Hdxt 750W MR machine. MRI showed asymmetrical hyperintensities in globus pallidus (Figure 1) and small punctuate hyperintense lesion in right cerebral hemisphere (Figure 2).

\section{DISCUSSION}

Dysplastic white matter lesions (DWMLs) are hyperintense foci on T2 and FLAIR sequences of MRI in patients with NF1. $(1,5)$ They are usually multiple and less than $2 \mathrm{cms}$ in size and are typically located in globus pallidus, brainstem, centrum semiovale, thalamus, internal capsule, corpus callosum, and cerebellum. $(1,3,5)$ DWMLs occur due to disorderly myelin metabolism resulting in early edema, vacuolization (Spongiform changes - Hyperintensity on T2 and FLAIR) followed by degeneration of neurons, so as the time progresses these hyperintense lesions disappear. $(1,3-5)$ Lesions in general are clinically silent, Goh etal in their study showed that patients with these lesions tend to have

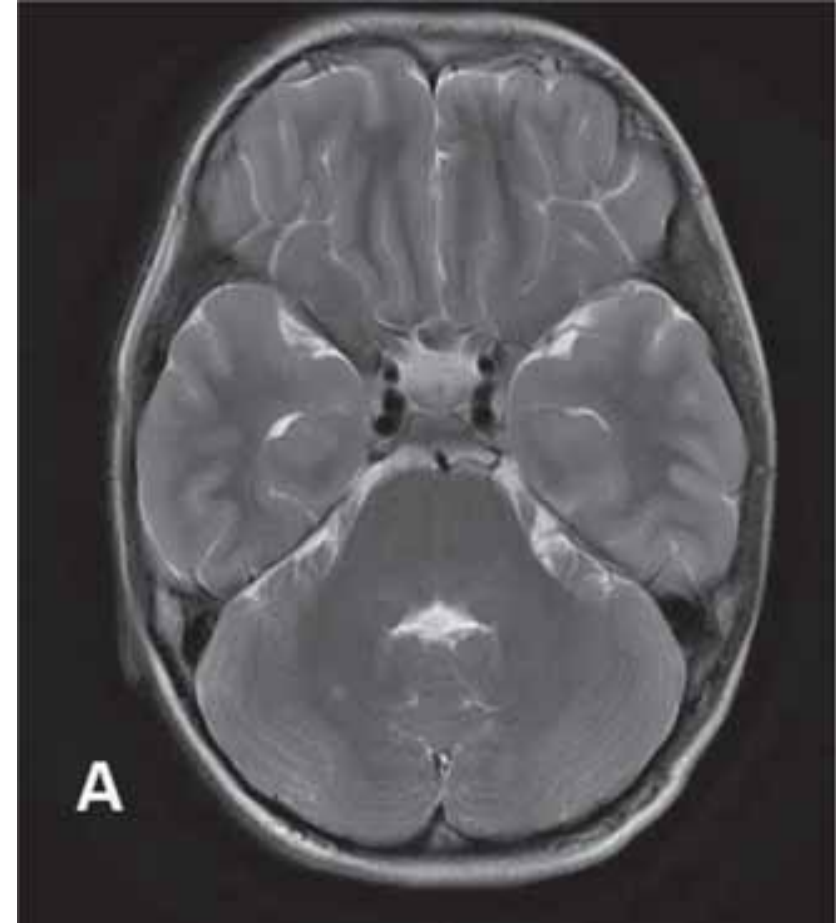

FIGURE 2. Axial T2W image of cerebellum showing small punctate T2 hyperintense lesion in the right cerebellum

reduced attention span. (9) Moore etal showed neuropsychological functioning of these lesions depends upon region involved and they proposed that whenever thalamus is involved patients have greater intellectual impairment. Involvement of the basal ganglia, brainstem and cerebellum are asymptomatic. (10) On imaging, these lesions are mildly hyperintense on $\mathrm{T} 1$ and do not show mass effect or contrast enhancement. (1) Neuronal destruction causes increased diffusivity and apparent diffusion coefficient values. $(7,11)$ Ferraz-Filho demonstrat-

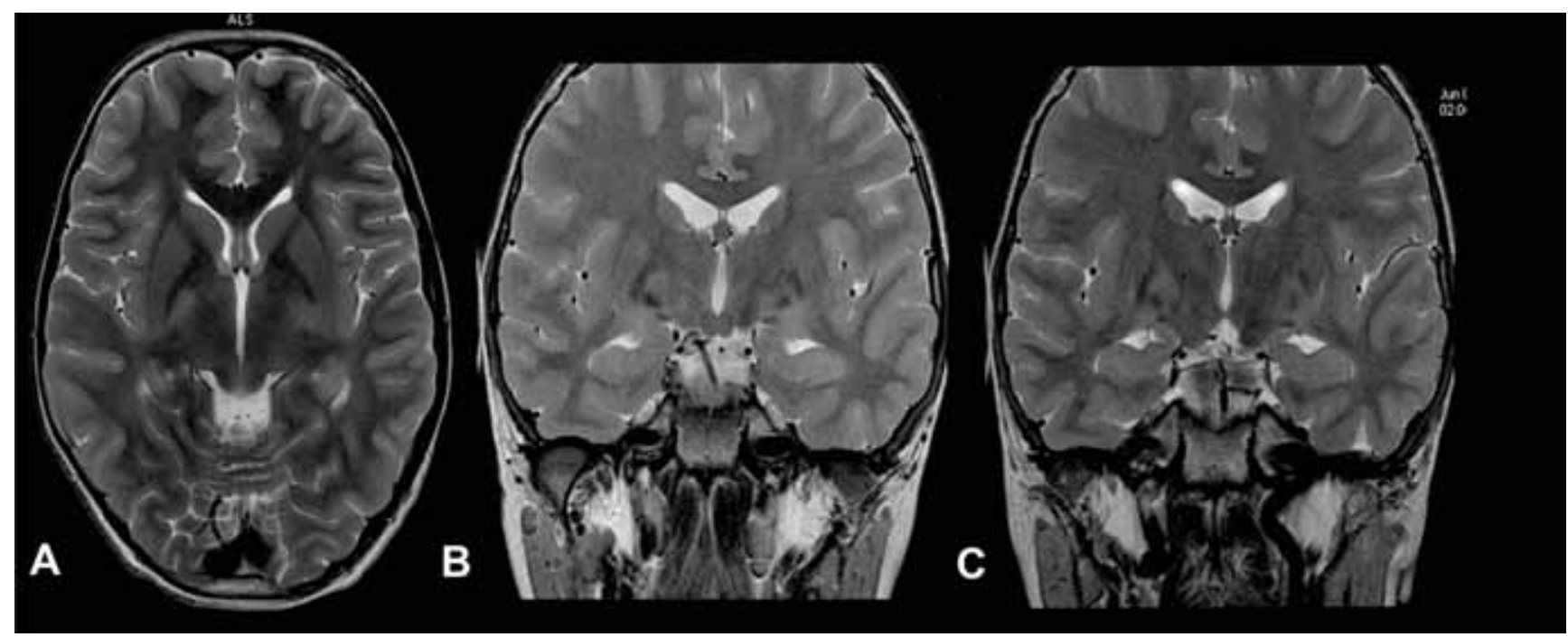

FIGURE 1. Axial and coronal T2 weighted MR images showing subtle asymmetrical T2 hyperintense lesions in globus pallidus replacing normal hypointensity 


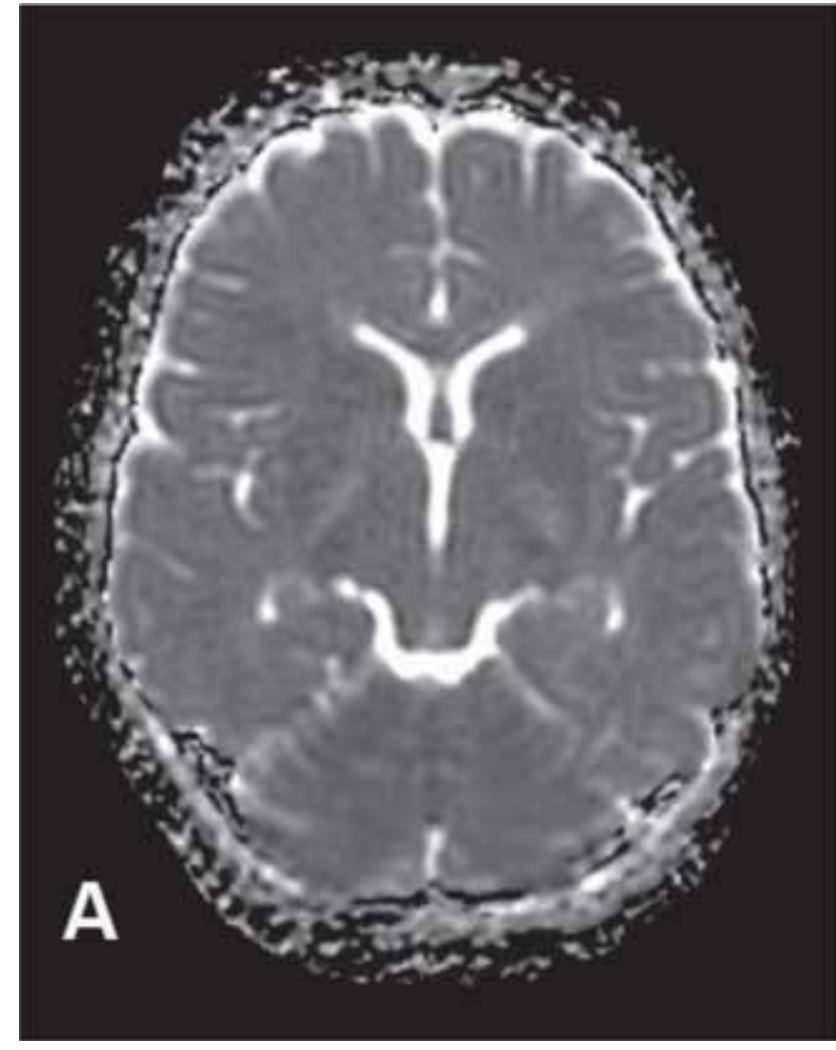

FIGURE 3.

ed fractional anisotropy abnormality in areas suggesting microstructural damage. (6) MR spectroscopy shows normal choline levels which helps us differentiate from gliomas. (12) Spontaneous regression of this DWML have been documented by age of twenty. Caution is advised not to confuse these lesions with malignant lesions like gliomas as biopsies from these lesions showed benign etiology. Khan et al have demonstrated no significant change in these lesions despite of clinical worsen- ing of the patients. (1) Lack of mass effect \& contrast enhancement are features of these lesions. NF1 lesions should be kept as differential for any hyperintense lesion in basal ganglia as commonly these lesions can be easily mistaken for other pathologies like viral encephalopathies and mitochondrial disorders. Parental counseling regarding the prognosis is very important to alleviate unnecessary apprehension. Interval follow-up is advised for large lesions causing mass effect, showing contrast enhancement or when lesions are located in optic pathway.

\section{CONCLUSION}

Dysplastic white matter lesions are due to spongiform myelinopathy of neurons, they appear as high signal intensity lesions on T2 and FLAIR sequences. Peak age of incidence is prepubertal age, generally they undergo spontaneous regression by age of 20. Lesions sometimes can be so subtle that they can be missed on imaging by radiologists, so they should be extremely cautious in reporting brain scans of patients with neurofibromatosis. Careful observation of characteristic locations of dysplastic white matter lesion can help in all such cases. Caution is advised in interpretation of these lesions as they can be mistaken for ischemic, infectious or neoplastic pathologies especially when history is masked. In doubtful cases radiological signs like lack of mass effect, contrast enhancement and MR spectroscopy can help differentiating these lesions from other causes. Parental counseling and assurance is advised in most cases with few cases requiring follow up imaging.

\section{REFERENCES}

1. Khan A., Beri S., Baheerathan A., Balki A., Hussain N., Gosalakkal J. Globus pallidus high-signal lesions: A predominant MRI finding in children with neurofibromatosis type 1. Ann Indian Acad Neurol 2013; 16:53-56.

2. DiPaolo D.P., Zimmerman R.A., Rorke L.B., Zackai E.H., Bilaniuk L.T., Yachnis A.T. Neurofibromatosis type 1: pathologic substrate of high-signal-intensity foci in the brain. Radiology 1995; 195:721-724.

3. Lin D.D.M., Barker P.B. Neuroimaging of phakomatoses. Semin Pediatr Neurol 2006; 13:48-62.

4. Gutmann D.H., Parada L.F., Silva A.J., Ratner N. Neurofibromatosis type 1: modeling CNS dysfunction. J Neurosci 2012; 32:14087-14093.

5. Choudhary A., Sharma S., Sankhyan N., Gulati S., Kumar A. Unidentified bright objects in neurofibromatosis type 1. Indian J Pediatr 2010; 77.

6. Ferraz-Filho J.R.L., José da Rocha A., Muniz M.P., Souza A.S., Goloni-Bertollo E.M., Pavarino-Bertelli E.C. Unidentified bright objects in neurofibromatosis type 1: conventional MRI in the follow-up and correlation of microstructural lesions on diffusion tensor images. Eur J Paediatr Neurol 2012; 16:42-47.

7. Eastwood J.D., Fiorella D.J., MacFall J.F., Delong D.M., Provenzale J.M., Greenwood R.S. Increased brain apparent diffusion coefficient in children with neurofibromatosis type 1. Radiology 2001; 219:354-358.

8. North K., Joy P., Yuille D., et al. Specific learning disability in children with neurofibromatosis type 1: significance of MRI abnormalities. Neurology 1994; 44:878-883.

9. Goh W.H.S., Khong P.L., Leung C.S.Y., Wong V.C.N. T2-weighted hyperintensities (unidentified bright objects) in children with neurofibromatosis 1 : their impact on cognitive function. $J$ Child Neurol 2004; 19:853-858.

10. Moore B.D., Slopis J., Schomer D., Jackson E., Levy B. Neuropsychological significance of areas of high signal intensity on brain MRIs of children with neurofibromatosis. Neurology 1996; 46:1660-1668.

11. Alkan A., Sigirci A., Kutlu R., et al. Neurofibromatosis type 1: diffusion weighted imaging findings of brain. Eur J Radiol 2005; 56:229-234.

12. Castillo M., Green C., Kwock L., et al. Proton MR spectroscopy in patients with neurofibromatosis type 1: evaluation of hamartomas and clinical correlation. AJNR Am J Neuroradiol 1995; 16:141-147. 